\title{
A Review on Poly-Lactic-Co-Glycolic Acid as a Unique Carrier for Controlled and Targeted Delivery Drugs
}

\author{
K. Prakash Raj ${ }^{1}$, K. Kathiresan², P. Pandian ${ }^{3}$ \\ 1,2,3 Department of Pharmacy, Annamalai University, Annamalainagar, Tamil Nadu, India.
}

\section{ABSTRACT}

In regulated and targeted drug distribution, biodegradable polymers have played a significant portion. Poly-lactic-co-glycolic acid (PLGA) has been an important desirable polymer in tissue engineering to meet a new drug delivery system. PLGAs, show a broad spectrum of erosion cycles and have tuning mechanical characteristics. Poly-lactic-co-glycolic acid (PLGA) has been the most successful polymeric biomaterial for use in controlled drug delivery systems. PLGA has been extensively studied, in particular, in the production of equipment for controlled distribution in industrial and research applications of small molecules, protein, and other macromolecules. PLGA is biocompatible and biodegradable, exhibits a wide range of erosion times, has tunable mechanical properties and most importantly, is a FDA approved polymer.

PLGA has many properties such as controlled and sustained release, low cancerinducing, long-standing biomedical applications, biocompatibility with tissues and cells, and prolonged residence time. It is otherwise called as 'Smart Polymer' because improvements are fragile to conduct PLGA that has been widely examined in industrial and academic applications to produce instruments for the target delivery of tiny molecular drugs, proteins, and other large molecules. An introduction about the chemistry, physicochemical properties, manufacturing techniques of the devices, toxicity, and the reason influencing their decrease and release of the drug was given in the present study. Mathematical modelling is a useful tool for identifying, characterizing, and predicting the mechanisms of controlled release.

Mathematical modelling applied against the target from PLGA - the devices has been clarified by discussing in the review, by explaining the underlying mathematical models and how this is used.

\section{KEY WORDS}

Biodegradable Polymers, PLGA, Biodegradability, Macromolecules
Corresponding Author:

Dr. P. Pandian,

Associate professor

Department of Pharmacy,

Annamalai University,

Annamalainagar - 608002,

Tamil Nadu, India.

E-mail: pandian0071@rediffmail.com

DOI: $10.14260 / j e m d s / 2021 / 416$

How to Cite This Article:

Raj KP, Kathiresan K, Pandian P. A review on poly-lactic-co-glycolic acid as a unique carrier for controlled and targeted delivery drugs. J Evolution Med Dent Sci 2021;10(27):2034-2041, DOI: 10.14260/jemds/2021/416

Submission 10-12-2020,

Peer Review 09-05-2021,

Acceptance 15-05-2021,

Published 05-07-2021.

Copyright (C) 2021 K. Prakash Raj et al. This is an open access article distributed under Creative Commons Attribution License [Attribution 4.0 International (CC BY 4.0)] 


\section{BACKGROUND}

Poly D, L-lactic-co-glycolic acid is one of the successful polymers used to make drugs, for example, drug carriers (DC). The Food and Drug Administration's (FDA) biocompatibility and biodegradation is one of the several polymers that has been approved for human use.1,2 Recent literature claims that the degradation of PLGA can be used without surgical interventions for the continuous release of drugs at desirable doses. In addition to that, the physical properties of a polymerdrug matrix can be modified to guarantee the optimal dosage and release time, based on the type of drug, by controlling the required parameters, such as molecular polymer weight, lactide to glycolide ratio, and drug concentration. However, a thorough evaluation was needed for the prospective side effects from dose increasing, unpredictable release, and polymer-drug interaction in this present study of PLGA in the industrial use, scale-up, and research of various drugs, proteins, and other macromolecules as delivery systems. They are used for various therapeutic applications such as cancer treatments or vaccinations, neurological conditions, inflammation, and other diseases. ${ }^{3}$ In applications for drug delivery, we also have potential advice on possible PLGA uses. Finally, existing developments in the growth of PLGA structures, including traditional and innovative functionalization techniques, will also be reviewed.

\section{Chemistry of PLGA}

PLA and PGA copolymers are poly-lactic acids. Polyester is a poly-lactic acid. Poly-lactic acid contains a standard D or L type asymmetric alpha carbon on rare occasions. Poly-D-lactic acid and poly-L-Lactic acid are the enantiomeric versions of the polymer. The PLGA is typically a poly - D term, with a corresponding proportion of the lactic - glycolic acid mixture and the D - and L - lactic acid types.

\section{Synthesis of PLGA}

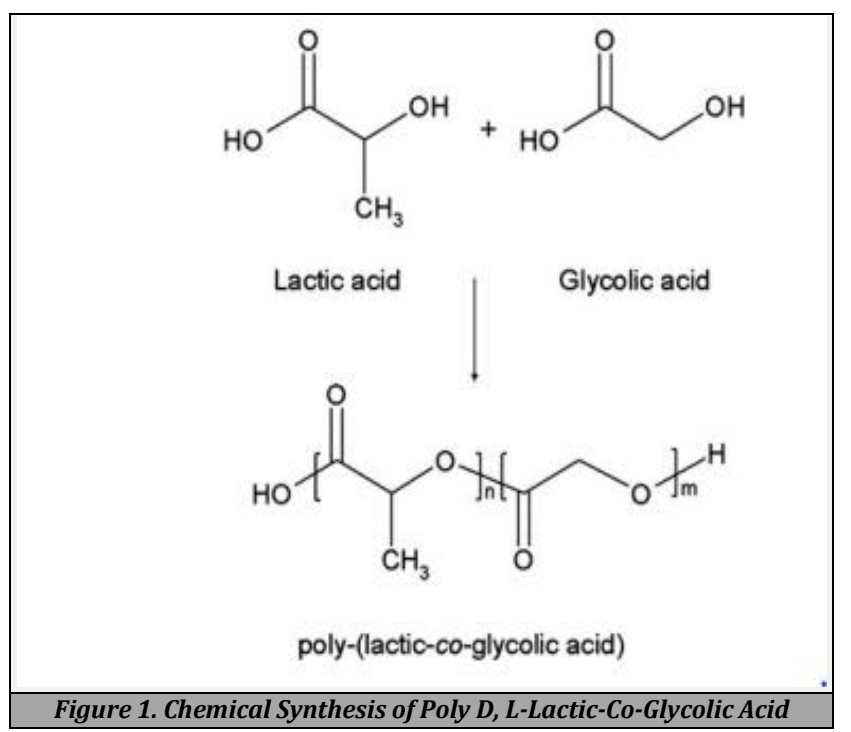

PLGA was synthesized in various quantities between its component monomers, lactic acid and glycolic acid . PLGA is produced from different lactide to glycolide ratio types (Fig1): these types were classified and generally with concerning monomer ratios, i.e., 75 percent lactic acid, 25 percent glycolic acid, and copolymer PLGA 75:25). Several synthesis processes were used to get PLGA, and process variables have a significant effect on the end product's physicochemical features. ${ }^{4,5}$

\section{Physicochemical Variabilities}

In physical, chemical properties, optically active PDLA and PLLA are almost equivalent. Polymer PLA is generally highly crystalline or completely amorphous through disordered polymer chains. PGA has a strong crystalline form and has a greater identity over the methyl group than the PLA. The solution is used in a large variety of solvents, including chlorination, acetone, tetrahydrofuran, and ethyl acetate. ${ }^{6}$ The changes in PLGA characteristics control drug release during polymer biodegradation as well as the integrated degradation rate. PLGA's physical properties, along with initial molecular weight, glycolide ratios, device size, water exposure to surface shape, and storage temperatures, have shown themselves to depend upon different variables. ${ }^{7}$

The PLGA copolymers are stated to be higher than $37^{\circ} \mathrm{C}$ and therefore glassy, with a highly stable chain structure. ${ }^{8}$ The copolymer TG is less effective than the physiology. Because of the absence of GA in the methylene carbonyl group (as opposed to LA), the PLGAs are amorphous copolymers.

\section{Biodegradation}

PLGA is degraded by its polyester backbone's hydrolytic cleavage. The kinetics of degradation of PLGA were characterized by data collection through size reduction column and continuous by modeling. ${ }^{9}$ The characterisation of polymer deficiency increases and the pseudo-first-order kinetics in Eq. 1. X was an increase in copolymer binding cleavage and time, as well as an initial average weight of molecules. $X$ was determined by calculating the initial average number of molecular weights and the average number of molecular weights per size exclusion chromatography at degradation time $t$.

$$
\ln (X)=\text { intercept }-k_{d^{t}}(E q .1)
$$

An acid can catalyse the hydrolysis of polyester. As products from hydrolysis of PLGA polymers are part of the carboxylic acid chain, the degradation of the polymer can lead to autocatalysis. ${ }^{10}$ The body metabolizes non-toxic chemicals that can be degraded by glycolic and lactic acid. ${ }^{11}$ Hydrolysing its ester bonds, PLGA degrades in aqueous environments by bulk or heterogeneous erosion. Parameters that are usually called invariant representations of a PLGA are due to the hydrolysis of PLGA. Four steps can be involved during its degradation such as hydration, initial degradation, constant degradation, and solubilisation. ${ }^{12}$

\section{Copolymer of PLGA}

The creation of different types of polyester block copolymers with polyethylene glycol has resulted in the administration of various methods in need of drug incorporation in the increased formulation for delivery. PLGA / PEG block copolymers from two ABA (PLGA) ${ }^{13}$ and BAB (PEG - PLGA - 
PEG) ${ }^{14}$ forms were processed as diblock (PLGA - PEG) ${ }^{15,16}$ or triblock molecules.

\section{Fabrication Techniques for PLGA}

PLGA has more attention in pharmaceutical industries to deliver drugs and proteins in a controlled or targeted manner. Due to drugs' biocompatibility, inappropriate kinetics for breaking down organic matter and mechanical compatibility, PLGA features will be conveniently processed and manufactured in different shapes and sizes. ${ }^{17}$ This division includes the different PLGA - controlled drug delivery device manufacturing techniques.

\section{Microparticle Preparation Techniques}

Solvent Evaporation Method - Single Emulsion Method

An immiscent, volatile organic solvent e.g., dichloromethane in water dissolved to a sufficient extent is first necessary to prepare a single-phase solution. The disbursed polymer-drug mixture solution was then emulsified in the presence of a PVA emulsifier in large volumes of water at the appropriate turbulent temperature. The organic solvent is then allowed to evaporate or dissolve in suitable conditions to solidify the oil droplets.

Finally, the emulsion is passed into a large amount of water or another tapered agent to diffuse the liquid associated with oil droplets. It was often used to disperse the solvent. The microspheres are then washed and dried under suitable conditions to produce a final injectable microsphere formulation ${ }^{18-21}$

\section{Double Emulsion Method}

An appropriate quantity of the drug substance is dissolved in the aqueous (deionized) process first. Then the drug solution is used to produce an emulsion of water-in-oil for the organic stage containing DCM - or PLA - solution. Next, in addition to an aqueous PVA solution, primary water-in-oil emulsion is applied and then emulsified for around one minute under the necessary tension mix conditions. It is then permitted to evaporate the organic solvent as emulsion techniques for $\mathrm{o} / \mathrm{w}$ type. Finally, the PLGA microspheres are available. ${ }^{19-21}$

\section{Phase Separation Method}

Coacervation is the division into two immiscible fluid states of the macromolecular solution, a dense coacervate phase, and a dilute equilibrium. This process is called simple coacervation in the presence of one macromolecule. This process is known as complex coacervation when two or more macromolecules are added together. Both processes are based on the idea of reducing the polymer's solubility in the organic phase to impact the polymer-rich phase formulation called coacervates. $22-25$ In this process, the polymer has been dissolved first in the requisite organic solvent and then dispersed into an aqueous solution in the necessary ratio. By modifying the solution conditions, phase separation is achieved. The droplet's size can be regulated by the system's different stirring rate and temperature. Washing, sieving, filtration, centrifugation, or freeze-drying can gather the final shape of the microspheres.
Spray Methods

Spray methods are rapid and convenient with few processing conditions and may be ideal for commercial, flexible treatment. There are two types of spray in the preparation of microspheres, such as spray congealing and spray drying. The fundamental concept behind these methods is to dry the drug and polymer in the air. In this spray drying process, the polymer has been dissolved first in the required volatile organic solvent.

The drug is then dispersed into the homogenization solution and then poured into the hot air stream. The mist or particles thus produced are in the range of $1-100 \mu \mathrm{m}$. The microspheres are shielded by means of a cyclone separator, while the solvent traces are removed by drying with vacuum. The cooling of the polymer solution can rely on spray congealing, while drying depends on the removal of the solvent. This technique may produce uniform double-walled microspheres. For batch and bulk production, it is a suitable technique. ${ }^{26}$

\section{Nanoparticulation}

Different methods were given for the active preparation of PLGA nanoparticles. The methods used to manufacture PLGA nanoparticles were taken into the adjustment of process parameters. The emulsion solvent method is the most common process for preparing solid, polymeric nanoparticles. But this strategy is mainly used in the hydrophobic drugs encapsulation. ${ }^{22}$ Nanoprecipitation methods can synthesize nanoparticles as well. The acetone is the dissolved polymer and medication and a water-based solution containing the addition of Pluronic F68. At acceptable temperatures and lower pressures, acetone evaporated, leaving behind polymer encapsulated nanoparticles with drugs. ${ }^{27}$

A polymer, solvent (non-chlorinated as acetone), containing water emulsion in oil, first designed as a mechanism of salting off: salt (e.g. tetrahydrous magnesium), stabilisation of oil. Water is used to diffuse acetone into it, resulting in nanoparticle formulations, until the quantity is sufficient.

Implant Preparation Techniques - Solvent Casting and Compression molding

The above one can be inserted or pumped for a long-term medication for formulating a macroscopic millimetre scale. ${ }^{28}$ one or to be a drug withhold that can supply for a longer amount of time. A drug and polymer mixture are to be dissolved in the desired proportion in a typical mixture (e.g., acetone) in this process, and to cast the mixture at about 600 $\mathrm{C}$ for complete dryness at about $800 \mathrm{C}$ at 25,000 psi to a final state of $1 \mathrm{~g} / \mathrm{cc}$, the substance of the solvent cast is then constricted into perfect geometry. It is the potential to inject this implant subcutaneously into the system. The purpose of this system against micro / nanospheres is that implants are reusable in warehouse mechanisms and cannot handle the harmful effects.

\section{Extrusion}

The polymer's drawing is a persistent cycle for the drug to blend in a fixed die for implants without the solvent to use. This happens when there is solvent casting expulsion. The 
method requires a mixture of extruders and polymer drugs with the appropriate small-size feed content. The polymer mixture was moved into a half-liquid state by means of heating, heating and surface giving due to the heating elements. The screw moves into the die with the mixture. This extrudate is then refrigerated and solidified until cut to the lengths needed for implants or other applications. ${ }^{29}$

Miscellaneous Systems - Multidrug Delivery Devices

The persistent may lead to a reduction in the tolerance of the recipient, supported through a pulsed drug release profile. New multi-pulsatile supply systems are built-in, which are quick and intermittent drug release accompanies a set off-loop time before the system has deteriorated. It has also demonstrated that these instruments can release various drugs over a cycle. PLGA is an attractive provider of devices for multidrug distribution and multiplication operations because of its favourable and smooth functions. ${ }^{30-33}$ These systems may be generalized to ensure that multiple drugs such as PLGA microchips are administered on a scheduled basis in a fixed range of pulse.

\section{Supercritical $\mathrm{CO}_{2}$}

In the recent period, the supercritical $\mathrm{CO} 2$ manufacturing methods with foaming agents have restricted some limitations, including solvent-casting and particulate leaching techniques, resulting from traditional methods of microporous foam forming. Usually, traditional methods require more purification steps to remove the residual solvent. For this case, more quantities of organic solvent is needed. Organic solvents can be reduced or removed from PLGA foams manufacturer by using supercritical $\mathrm{CO} 2$ as a foaming agent. Encapsulated with polymer, the drug / protein is generated using emulsion techniques. A solvent is quickly inserted to use this technique into a $\mathrm{CO} 2$ cell after the emulsion. PLGA's transitional temperature is reducing under high pressures, resulting in a liquid polymer that dissolves CO2. The pressurization and depressurization within the liquid polymer, the clustering of CO2 molecules are thermodynamic instability. The emulsion results in a porous polymer structure as it leaves $\mathrm{CO2.34,35}$ Greater drug release reported due to the higher surface to volume ratios for microporous foams.

\section{Multifunctional PLGA Micro / Nanoparticles}

PLGA micro-nanoparticles were often used in a single formulation for different applications. ${ }^{36} \mathrm{~A}$ few multifunctional particles of material components have been formulated to minimise the unwanted effect of the incarnated substance. These particulate matters may not only act as a transport of the embedded drug but can decrease undesirable side effects by targeted drug delivery. However, before a final structure is calculated, the chemical reactivity of these adjutants ${ }^{37}$ has to be assessed. The emulsion techniques usually complete the development of these multifunctional particles.

\section{Release of Drugs from PLGA Carriers}

The release of drugs from PLGA transports is primarily dependent on copolymers' hydrolysis or biodegradation. The processes involved in PLGA - copolymers degradation include mass diffusion, surface diffusion, bulk erosion, and surface erosion. The release of drugs from this carrier is biphasic in nature. 38,39

1. The initial explosion in drugs is associated with drug structure, drug concentration, and hydrophobicity in polymers. The drug on the surface in contact with the medium releases itself in compliance with the solubility and water penetration into the polymer matrix. Random splitting of PLGA reduces molecular polymer weight considerably, but there is no considerable weight loss or soluble monomer substance.

2. The prescription is released progressively through the thicker depleted sheet in the second level. The polymer hydrolysed into soluble monomeric and oligomeric elements in the matrix. It enables the release of a medicine passage by erosion and diffusion before complete solvents of polymers. The class of drugs also has a significant role in matrix drawing up the aqueous process.

The function of the enzymes is unclear in any PLGA biodegradation. However, some researchers proposed that the PLGA breakdown could play an enzymatic role based on the disparity of the degradation rate in vitro and in vivo.

\section{Factors Affecting Drug Release}

Since many variables can impact the degradation process, the release rate trend is always erratic. Different parameters may affect the deterioration rates, understand the factors that cause PLGA degradation and build a drug delivery system.

- Molecular weight: Molecular weight rises from a typical PLGA 10 to $20 \mathrm{kDa}$; decay is recorded for several weeks up to several months. ${ }^{40}$

- GA / LA ratio: PLGA's higher LA degree is lower. Since hydrophilic consumes less water than slowly decreases due to methyl lateral groups; as a result, PLA has a hydrophobic role in the methyl lateral group than PGA. ${ }^{41}$ A reasonable exception to this exception is the law of the 50:50 copolymer, which indicates quicker degradation;

- $\quad$ Stereochemistry: Lactic acid mixtures D and L are used more commonly in PLGA manufacturing because their water rate in the amorphous $\mathrm{D}, \mathrm{L}$ areas penetration is higher, contributing to accelerating PLGA degradation. ${ }^{41}$

- The end group functionalisation: Polymers end-capped with esters (compared to carboxylic acid) exhibit longer degradation half-lives. Furthermore, the unit structure dramatically affects PLGA degradation behaviour, which depends on the water accessibility. Furthermore, the underlying acidic media speeds up the oxidation of PLGA by autocatalysis. ${ }^{42}$

- Crystallinity: Copolymer structure is also influenced by significant properties like crystallinity and glass transition temperature, indirectly influencing the degradation rate. Crystallinity effect tests are currently being conflictingly investigated at the deterioration scale. Few groups ${ }^{43,44}$ suggested that the rate of degradation of the lactic acid crystallinity (PLLA) is increased due to the increasing hydrophilicity of the semi-crystalline polymer.

- The PH: The polymer deterioration are increased by invitro biodegradation / hydrolysis of PLGA in acidic and 
alkaline media. The disparity of both acidic and neutral media has less capability, however, because of the carboxylic end - group autocatalysis. ${ }^{45}$

\section{PLGA Toxicity}

Bakhaidar et al. ${ }^{46}$ PLGA toxicity has been studied. ${ }^{46}$ Here, the investigators analysed the effects of PLGA - PEG nanoparticles on aggregation and platelet activation. The findings suggest that nanoparticles of all dimensions are connected to the platelet's surface and theoretically internalized. Furthermore, the NP - platelet relationship has shown the lack of a platelet aggregation, which makes these PLGA Nano formulations promising structures for supplying targeted medication to platelets. PLGA product toxicological tests demonstrate that local tissue reactions can occur at the site of application. ${ }^{47,48}$ Several studies have indicated that nanoparticles can produce relevant biological distribution and adverse effects profiles. 49

\section{Drug Release Profile for PLGA Modelling}

Mathematical modelling is a valuable method for defining to find out the best mechanism and experimental process involved in the parameters and minimizing the error. ${ }^{50,51}$

In the fitting of controlled release data, mechanistic models may build as predictive differential equation systems that explain the mass transport phenomenon that regulates the release of drugs and due to their more excellent predictive capabilities over empirical models. In PLGA release systems, both the models, such as mechanistic and empirical, are applied. PLGA's early mechanistic modelling concentrated emphasis on characterizing the polymer lower processes combined with drug entrapment. So in the additional account, the degradation process was revised in kinetics and its characteristics.

Due to time-dependent permeability, the incorporated Higuchi equation was updated to account for PLGA polymer matrix consequences in the degradation.

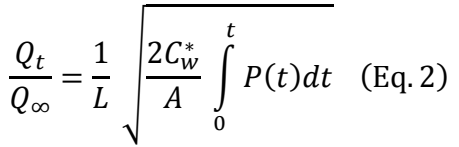

Where Qt is the total mass of the release region of each drug unit at time $t, \mathrm{C}^{*} \mathrm{w}$ is the solubility release media's of the drug, $\mathrm{P}$ are duration depending on permeability, $\mathrm{A}$ is the loading of a drug, $\mathrm{L}$ is half of the matrix's thickness, and Qinf are released area loading of drug in unit. ${ }^{52}$ The PLGA release device has to change the porosity in the model, which has benefit into the account, and the PLGA particles are scanned in an electron microscope for degrading. The coefficient of PLGA release was not enough through the Higuchi model to assume the constant permissibility.

Fick's second law of spherical geometry is based upon a weight shift in molecular polymers.

$$
M_{t}=4 \pi r^{2}\left[\sqrt{2\left(C_{0}-C_{s}\right) C_{s} D t}+\frac{4 C_{s} D t}{9 r}\left(\frac{C_{s}}{2 C_{0}-C_{s}}-3\right)\right] \text { (Eq. 3) }
$$

Where $M_{t}$ was mass released cumulative time $t, r$ is the radius of the microparticle, $\mathrm{C}_{0}$ is the initial drug concentration within the polymer matrix, $\mathrm{C}_{s}$ is the solubility of the drug in the medium, D are coefficient diffusion. ${ }^{53}$ The diffusion coefficient is accounted for by the changing molecular weight in this model and the models are limited in spherical particle format. The diffusion coefficient is accounted for by the changing molecular weight in this model.

However, for non-spherical system morphologies, this model is limited to the spherical particle format and must be resolved.

The released data from molecules suggest that the Eq.4 Model for the released mechanism is Roseman-Higuchi.

$(1-F) \ln (1-F)+F=\frac{4 C D_{t}}{A a_{0}^{2}}$ (Eq. 4$)$

Ao are radius cylinders. The $\mathrm{C}$ are solubility in the substance of polymer, D is the diffusiveness, and A is the original overall quality of the product in polymer ${ }^{54} \mathrm{~F}$ is the proportion of the product release at time t. This model could be helpful for PLGA formulations that degrades significantly below the standard 50:50 PLGA formulation used because polymer degradation in these cases would have less impact on release. 55

It was found however that the Higuchi models performed little after this model was ready to release results.

$\frac{d M_{t}}{d_{t}}=C \sqrt{\frac{\exp (k t)}{t}}$ (Eq. 5)

From the microsphere of PEG - PLGA - PEG triblock copolymers, the Heller equation was unaffected by small, uncontrollable hydrophobia release molecule from the microsphere. The first parameters are a coefficient of permeability and the initial concentration in this model C. 56

$\frac{d M_{t}}{d t}=B \exp (-k t)+C \sqrt{\frac{\exp (k t)}{t}}(E q .6)$

$\mathrm{B}$ and $\mathrm{k}$ are diffusion coefficients of the initial drug concentration of the device thickness. ${ }^{57}$ A spherical drug was released to show the revolutionary release of the system composed of an advanced PLGA disc model and the controlled release of polymer degradation from the surface (Eq. 7).The first order was surface dissolution was defined as the burst phase. In the release of burst, equations are helpful in contributing to major released factors.

$$
F_{\text {tot }}=F_{B}\left(1-\exp \left(-K_{B} t\right)\right)+\left(1-F_{B}\left(\frac{\exp \left(k t-k T_{\max }\right.}{1+\exp \left(k t-k T_{\max }\right)}\right)\right.
$$

FTOT is the cumulative portion of the prescription released at $\mathrm{t}$, the frequency constant is $\mathrm{Kb}$, and the rate constant is $\mathrm{K}$, and the time for maximum release is Tmax. For the release of PLGA drugs, the classic diffusion model of Fickian has been modified so that a time-dependent diffusion coefficient was introduced based on the molecular degradation of the pole. (Eq. 8-10)

$\frac{M_{t}}{M_{\infty}}=1-\frac{6}{\pi^{2}} \sum_{n=0}^{\infty}\left[\frac{1}{n^{2}} \cdot \exp \left(\frac{-D n^{2} \pi^{2} t}{r^{2}}\right)\right]$

$D=D_{0}+\frac{k}{M W}$

$M W=78.4 \exp \left(-k_{\mathrm{deg} r} t\right) \quad(E q .10)$

Mt is the accumulated mass emitted at t time, $\mathrm{M}_{\mathrm{inf}}$ is the infinite time cumulative mass released, $\mathrm{r}$ is the micro - radius, 
D is the coefficient of diffusion, MW is the molecular polymer weight, D0 is the first drug diffusiveness, $\mathrm{k}$ is constant, and kdeg is the primary polymer degrader rate constant. ${ }^{57}$ Recent mathematical simulation advances of controlled release schemes are equipped with numerical convergence deterministic predictive models such as PLGA for bulk erosion. ${ }^{58}$ Numerical convergence deterministic models were used. The PLGA released modeller was recently used to construct an internal Fickian diffusion model with a temporal porosity term, which represents a pseudo-first-order degradation of the PLGA polymer matrix.

$\frac{\partial C_{\Delta}}{\partial_{t}}=\nabla\left(D_{\text {eff }} \nabla C_{\Delta}\right) \quad($ Eq. 11$)$

$D_{\mathrm{eff}}=D \varepsilon(t) \quad($ Eq. 12$)$

$\varepsilon(t)=\frac{1}{2}\left[\operatorname{erf}\left(\frac{t-\bar{\tau}}{\sqrt{2 \sigma^{2}}}\right)+1\right]$

$\bar{\tau}=\frac{-1}{k C_{w}} \ln \left|\frac{M_{w t}}{M_{w o}}\right|$

The $\mathrm{Ca}$ are the matrix agent, $\mathrm{D}_{\text {eff }}$ is the effective diffusion coefficient, $\mathrm{D}$ is the diffusion coefficient, e is the porosity at $\mathrm{t}$, tau is polar formation meantime, $\mathrm{kCw}$ are pseudo-first-order degradation rate, $\mathrm{M}_{\mathrm{wo}}$ is the original polymer weight, $\mathrm{M}_{\mathrm{wr}}$ is an average polymer $\mathrm{m}$, and $\mathrm{M}_{\mathrm{wr}}$ is the average polymer molecular weight in a differential volume of the matrix that permits diffusion of the encapsulated agent. A wide range of therapeutically essential released behaviours have been successfully predicted by simulation of this model. Although complex models and methods of modelling have been developed, classical empirical links remain in most standard types in the modelling of PLGA release data. ${ }^{59,60}$

\section{CONCLUSIONS}

PLGA polymers have proved to be excellent carriers for controlled drug, peptide, and protein administration, owing to their biocompatibility and biodegradability. In this review, we also discussed the various factors that affected the degradation rate of PLGA and reported the detailed account of different techniques that were involved in the preparation of PLGA carriers. These systems address various shortcomings of traditional dosage forms that eventually enhance the compliance of patients and drug therapy. Apart from its applications in drug delivery technology, PLGA still finds valuable applications in biomedical sciences.

\section{Future Prospects}

In the future, the significance of PLGA as a biodegradable polymer is indicated by various PLGA - based delivery systems that have been documented or commercialized. PLGA is also regarded as the main polymer of production in the drug for engineering tissue application and - eluting medical devices, in addition to drug delivery applications. For example, to monitor the release of cardiovascular drugs, only a few PLGA nanoparticle-based stent devices are available. Similarly, PLGA
- based nanosystems have a great scope in the production of cosmeceutical products where the penetration of different active ingredients can be increased by enclosing them in the PLGA matrix. PLGA - PEG, PLGA - poloxamers, PLGA - calcium phosphate (PLGA - CaP) ceramics, and brushite - PLGA systems are some of these modified systems already developed. PLGA's non-toxic, non-immunogenic, biocompatible, and biodegradable activity provides promising prospects for its usefulness in designing future intelligent drug delivery systems that would find wide-ranging applications in the delivery of vaccines, drug targeting, and nanotheranostics.

Financial or other competing interests: None.

Disclosure forms provided by the authors are available with the full text of this article at jemds.com.

\section{REFERENCES}

[1] Makadia HK, Siegel SJ. Poly Lactic-co-Glycolic Acid (PLGA) as biodegradable controlled drug delivery carrier. Polymers (Basel) 2011;3(3):1377-97.

[2] Loureiro JA, Pereira MC. PLGA based drug carrier and pharmaceutical applications: the most recent advances. Pharmaceutics 2020;12(9):903.

[3] Guarecuco R, Lu J, McHugh KJ, et al. Immunogenicity of pulsatile-release PLGA microspheres for single-injection vaccination. Vaccine 2018;36(22):3161-8.

[4] Zhou SB, Deng XM, Li XH, et al. Synthesis and characterization of biodegradable, low molecular weight aliphatic polyesters and their use in protein-delivery systems. J Appl Polym Sci 2004;91(3):1848-56.

[5] Wang ZY, Zhao YM, Wang F, et al. Syntheses of poly (Lactic Acid-co-Glycolic Acid) serial biodegradable polymer materials via direct melt polycondensation and their characterization. J Appl Polym Sci 2006;99(1):244-52.

[6] Uhrich KE, Cannizzaro SM, Langer RS, et al. Polymeric systems for controlled drug release. Chem Rev 1999;99(11):3181-98.

[7] Houchin ML, Topp EM. Physical properties of PLGA films during polymer degradation. J Appl Polym Sci 2009;114(5):2848-54.

[8] Passerini N, Craig DQM. An investigation into the effects of residual water on the glass transition temperature of polylactide microspheres using modulated temperature DSC. J Control Release 2001;73(1):111-5.

[9] Kenley RA, Lee MO, Mahoney TR, et al. Poly (Lactide-coGlycolide) decomposition kinetics in vivo and in vitro. Macromolecules 1987;20(10):2398-403.

[10] Siepmann J, Elkharraz K, Siepmann F, et al. How autocatalysis accelerates drug release from PLGA-based microparticles: a quantitative treatment. Biomacromolecules 2005;6(4):2312-9.

[11] Siegel SJ, Kahn JB, Metzger K, et al. Effect of drug type on the degradation rate of PLGA matrices. Eur J Pharma Biopharm 2006;64(3):287-93.

[12] Engineer C, Parikh J, Raval A. Review on hydrolytic degradation behavior of biodegradable polymers from controlled drug delivery system. Trends Biomater Artif Organs 2011;25(2):79-85.

[13] Ghahremankhani AA, Dorkoosh F, Dinarvand R. PLGAPEG-PLGA triblock copolymers as an in-situ gel-forming 
system for calcitonin delivery. Polym Bull 2007;59(5):637-46.

[14] Jeong B, Bae YH, Kim SW. In situ gelation of PEGPLGAPEG triblock copolymer aqueous solutions and degradation thereof. J Biomed Mater Res 2000;50(2):171-7.

[15] Cheng J, Teply BA, Sherifi I, et al. Formulation of functionalized PLGA-PEG nanoparticles for in vivo targeted drug delivery. Biomaterials 2007;28(5):869-76.

[16] Li Y, Pei Y, Zhang X, et al. PEGylated PLGA nanoparticles as protein carriers: synthesis, preparation and biodistribution in rats. J Control Release 2001;71(2):20311.

[17] Houchin ML, Topp EM. Chemical degradation of peptides and proteins in PLGA: a review of reactions and mechanisms. J Pharm Sci 2008;97(7):2395-404.

[18] Arshady R. Preparation of biodegradable microspheres and microcapsules: 2. Polyactides and related polyesters. J Control Release 1991;17(1):1-21.

[19] Rosca ID, Watari F, Uo M. Microparticle formation and its mechanism in single and double emulsion solvent evaporation. J Control Release 2004;99(2):271-80.

[20] Sah H. Microencapsulation techniques using ethyl acetate as a dispersed solvent: effects of its extraction rate on the characteristics of PLGA microspheres. J Control Release 1997;47(3):233-45.

[21] King TW, Patrick CW Jr. Development and in vitro characterization of vascular endothelial growth factor (VEGF)-loaded poly (D, L-lactic-co-glycolic acid)/poly (ethylene glycol) microspheres using a solid encapsulation/single emulsion/solvent extraction technique. J Biomed Mater Res 2000;51(3):383-90.

[22] Mao S, Xu J, Cai C, et al. Effect of WOW process parameters on morphology and burst release of FITC-dextran loaded PLGA microspheres. Int J Pharm 2007;334(1-2):137-48.

[23] Thomasin C, Ho NT, Merkle HP, et al. Drug microencapsulation by PLA / PLGA coacervation in the light of thermodynamics. 1. Overview and theoretical considerations. J Pharm Sci 1998;87(3):259-68.

[24] Thomasin C, Merkle HP, Gander B. Drug microencapsulation by PLA / PLGA coacervation in the light of thermodynamics. 2. Parameters were determining microsphere formation. J Pharm Sci 1998;87(3):269-75.

[25] Edelman R, Russell RG, Losonsky G, et al. Immunization of rabbits with enterotoxigenic E. coli colonization factor antigen (CFA/I) encapsulated in biodegradable microspheres of poly (lactide-co-glycolide). Vaccine 1993;11(2):155-8.

[26] Berkland C, Pollauf E, Pack DW, et al. Uniform doublewalled polymer microspheres of controllable shell thickness. J Control Release 2004;96(1):101-11.

[27] Hans ML, Lowman AM. Biodegradable nanoparticles for drug delivery and targeting. Curr Opin Solid State Mater Sci 2002;6(4):319-27.

[28] Rabin C, Liang Y, Ehrlichman RS, et al. In vitro and in vivo demonstration of risperidone implants in mice. Schizophr Res 2008;98(1-3):66-78.

[29] Wang C, Wang W, Meyer RF, et al. A rapid method for creating drug implants: translating laboratory-based methods into a scalable manufacturing process. J Biomed Mater Res B Appl Biomater 2010;93(2):562-72.

[30] Yang $R$, Chen $T$, Chen $H$, et al. Microfabrication of biodegradable (PLGA) honeycombstructures and potential applications in implantable drug delivery. Sens Actuat B Chem 2005;106(2):506-11.

[31] Grayson ACR, Choi IS, Tyler BM, et al. Multi-pulse drug delivery from a resorbable polymeric microchip device. Nat Mater 2003;2(11):767-72.

[32] Grayson ACR, Cima MJ, Langer R. Size and temperature effects on poly (lactic-co-glycolic acid) degradation and micro reservoir device performance. Biomaterials 2005;26(14):2137-45.

[33] Stubbe BG, Smedt SCD, Demeester J. Programmed polymeric devices for pulsed drug delivery. Pharm Res 2004;21(10):1732-40.

[34] Koushik K, Kompella UB. Preparation of large porous deslorelin-PLGA microparticles with reduced residual solvent and cellular uptake using a supercritical carbon dioxide process. Pharm Res 2004;21(3):524-35.

[35] Davies OR, Lewis AL, Whitaker MJ, et al. Applications of supercritical $\mathrm{CO} 2$ in the fabrication of polymer systems for drug delivery and tissue engineering. Adv Drug Deliv Rev 2008;60(3):373-87.

[36] Xu JS, Huang J, Qin R, et al. Synthesizing and binding dualmode poly (lactic-co-glycolic acid) (PLGA) nanobubbles for cancer targeting and imaging. Biomaterials 2010;31(7):1716-22.

[37] Zhang L, Xu JS, Sanders VM, et al. Multifunctional microbubbles for image-guided antivascular endothelial growth factor therapy. J Biomed Opt 2010;15(3):030515.

[38] Ramchandani M, Robinson D. In vitro and in vivo release of ciprofloxacin from PLGA 50:50 implants. J Control Release 1998;54(2):167-75.

[39] Faisant N, Siepmann J, Benoit JP. PLGA-based microparticles: elucidation of mechanisms and a new, simple mathematical model quantifying drug release. Eur J Pharm Sci 2002;15(4):355-66.

[40] Wu XS, Wang N. Synthesis, characterization, biodegradation and drug delivery application of biodegradable lactic/glycolic acid polymers. Part II: biodegradation. J Biomater Sci Polym Ed 2001;12(1):2134.

[41] Lu LC, Peter SJ, Lyman MD, et al. In vitro degradation of porous poly (L-lactic acid) foams. Biomaterials 2000;21(15):1595-605.

[42] Holy CE, Dang SM, Davies JE, et al. In vitro degradation of a novel poly (lactide-co-glycolide) 75/25 foam. Biomaterials 1999;20(13):1177-85.

[43] Alexis F. Factors affecting the degradation and drugrelease mechanism of poly (lactic acid) and poly [(lactic acid)-co-(glycolic acid)]. Polym Int 2005;54(1):36-46.

[44] Tsuji H, Mizuno A, Ikada Y. Properties and morphology of poly(L-lactide). III. Effects of initial crystallinity on longterm in vitro hydrolysis of high molecular weight poly ( $\mathrm{L}-$ lactide) film in phosphate-buffered solution. J Appl Polym Sci 2000;77(7):1452-64.

[45] Zolnik BS, Burgess DJ. Effect of acidic pH on PLGA microsphere degradation and release. J Control Release 2007;122(3):338-44.

[46] Bakhaidar R, Green J, Alfahad K, et al. Effect of size and concentration of PLGA-PEG nanoparticles on activation and aggregation of washed human platelets. Pharmaceutics 2019;11(10):514.

[47] Dailey L, Jekel N, Fink L, et al. Investigation of the proinflammatory potential of biodegradable nanoparticle 
drug delivery systems in the lung. Toxicol Appl Pharmacol 2006;215(1):100-8.

[48] Sundback CA, Shyu JY, Wang Y, et al. Biocompatibility analysis of poly (glycerol sebacate) as a nerve guide material. Biomaterials 2005;26(27):5454-64.

[49] Olivier JC, Fenart L, Chauvet R, et al. Indirect evidence that drug brain targeting using polysorbate 80 -coated poly butyl cyanoacrylate nanoparticles is related to toxicity. Pharm Res 1999;16(12):1836-42.

[50] Klose D, Siepmann F, Elkharraz K, et al. PLGA-based drug delivery systems: importance of the type of drug and device geometry. Int J Pharm 2008;354(1-2):95-103.

[51] Göpferich A. Mechanisms of polymer degradation and erosion. Biomaterials 1996;17(2):103-14.

[52] Chiu LK, Chiu WJ, Cheng Y. Effects of polymer degradation on drug released-mechanistic study of morphology and transport properties in 50:50 poly (dl-lactide-coglycolide). Int J Pharm 1995;126(1-2):169-78.

[53] Faisant N, Siepmann J, Richard J, et al. Mathematical modeling of drug release from bioerodible microparticles: effect of gamma-irradiation. Eur J Pharm Biopharm 2003;56(2):271-9.
[54] Hsu Y, Gresser JD, Trantolo DJ, et al. In vitro controlled release of isoniazid from poly (lactide-co-glycolide) matrices. J Control Release 1994;31(3):223-8.

[55] Jeong B, Bae YH, Kim SW. Drug release from biodegradable injectable thermosensitive hydrogel of PEG-PLGA-PEG triblock copolymers. J Control Release 2000;63(1-2):155-63.

[56] Gallagher KM, Corrigan OI. Mechanistic aspects of the release of levamisole hydrochloride from biodegradable polymers. J Control Release 2000;69(2):261-72.

[57] Faisant N, Siepmann J, Benoit JP. PLGA-based microparticles: elucidation of mechanisms and a new simple mathematical model quantifying drug release. Eur J Pharm Sci 2002;15(4):355-66.

[58] Rothstein SN, Federspiel WJ, Little SR. A simple model framework for the prediction of controlled release from bulk eroding polymer matrices. J Mater Chem 2008;18(16):1873-80.

[59] Budhian A, Siegel SJ, Winey KI. Controlling the in vitro release profiles for a system of haloperidol-loaded PLGA nanoparticles. Int J Pharm 2008;346(1-2):151-9.

[60] Sun X, Xu C, Wu G, et al. Poly (Lactic-co-Glycolic Acid): applications and future prospects for periodontal tissue regeneration. Polymers (Basel) 2017;9(6):189. 